\title{
Exo-antigens from Paracoccidioides lutzii applied to immunodiagnosis of Paracoccidioidomycosis: an report of our experience in Brazil's Midwest
}

$\underline{\text { Simone Schneider Weber }}^{1,2^{*}}$; Karine Mattos ${ }^{1}$; Juliana Fernandes ${ }^{1}$; Edy Firmina Pereira ${ }^{1}$; Ana Paula Ramos Pereira ${ }^{1}$; Clayton Luiz Borges ${ }^{3}$; Ana Flávia Alves Parente ${ }^{4}$; Marilene Rodrigues Chang ${ }^{1}$; Rinaldo Pôncio Mendes ${ }^{5}$; Anamaria Mello Miranda Paniago ${ }^{1}$

${ }^{1}$ Faculdade de Medicina da Universidade Federal de Mato Grosso do Sul, Campo Grande, Mato Grosso do Sul, Brasil; ${ }^{2}$ Instituto de Ciências Exatas e Tecnologia, Universidade Federal do Amazonas, Itacoatiara, Amazonas, Brazil; ${ }^{3}$ Laboratório de Biologia Molecular, Instituto de Ciências Biológicas, Universidade Federal de Goiás, Goiânia, Goiás, Brazil; ${ }^{4}$ Universidade de Brasília, Brasília, DF, Brazil; ${ }^{5}$ Faculdade de Medicina de Botucatu, Universidade Estadual Paulista, Botucatu, São Paulo, Brazil.

\section{$\triangle$ simone.weber@ufms.br}

Abstract code : $\mathrm{P} 071$

In central-west region of Brazil, where Paracoccidioides lutzii predominates, has been described low reactivity in immunological tests using Paracoccidioides standard antigen (AgB-339). Here, we aimed to evaluate the performance of exo-antigens from $P$. lutzii against patient sera from Brazilian central-west region (CWR), and to speculate their influence under PCM immunological diagnose, as well sorological followup of these patients. The cell-free culture antigens, known as exo-antigens, were obtained from P. lutzii (ATCC MYA-826), defined as PlAg, and proved by double agar-gel immunodiffusion (DID) test against 53 PCM suspect serum samples from CWR. The PCM confirmation was given in $81.13 \%$ of cases (43/53) by direct exam, biopsy specimens or culture.
P. Lutzii yeasts (ATCC MYA - 826)

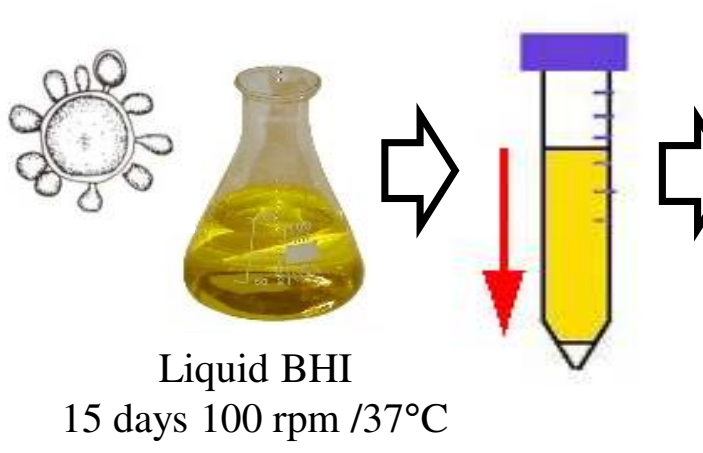

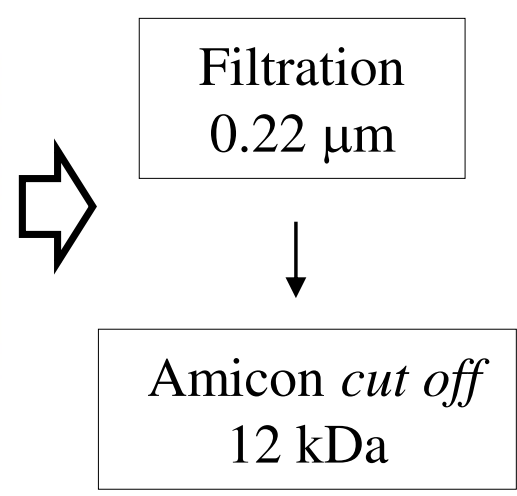

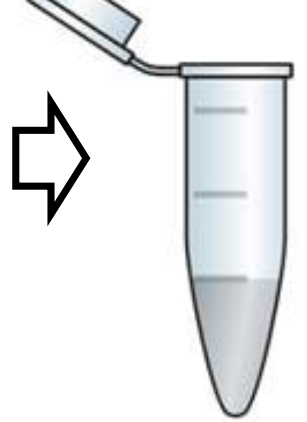

FIGURE 1. Metodological fluxogram of $P$. lutzii exoantigen obtention to use in sorological tests for antibodies anti-PCM detectation. The exoantigens species-specific were obtained from P.lutzii isolates (ATCCMYA-826).

The obtained Ag- $P l$ was validated by testing (ID) 43 serum samples from patients with confirmed PCM diagnosis (direct mycological, culture and/or biopsy positive results). All patients were tested using both exoantigens: the standard (Ag-339) and Ag- $\mathrm{Pl}$ (obtained in this study). Of these, $60.46 \%$ (26/43) was negative when tested with only AgB-339. Although the literature suggests high specificity and sensitivity to DID test (65 to 100\%), we able to find only $39.53 \% \quad(17 / 43)$ of reactivity using Paracoccidioides standard antigen. In the other hand, this percent increased to $72.09 \%$ (31/43), when we added PlAg on assay, highlighting its role to immunodiagnose.

\begin{tabular}{lr} 
ExoAg-Pl & $32.55 \%(14 / 43)$ \\
ExoAg-339 & $9.30 \%(4 / 43)$ \\
ExoAg-Pl / ExoAg-339 & $30.23 \%(13 / 43)$ \\
\hline TOTAL (+) & $\mathbf{7 2 . 0 9 \% ( 3 1 / 4 3 )}$
\end{tabular}

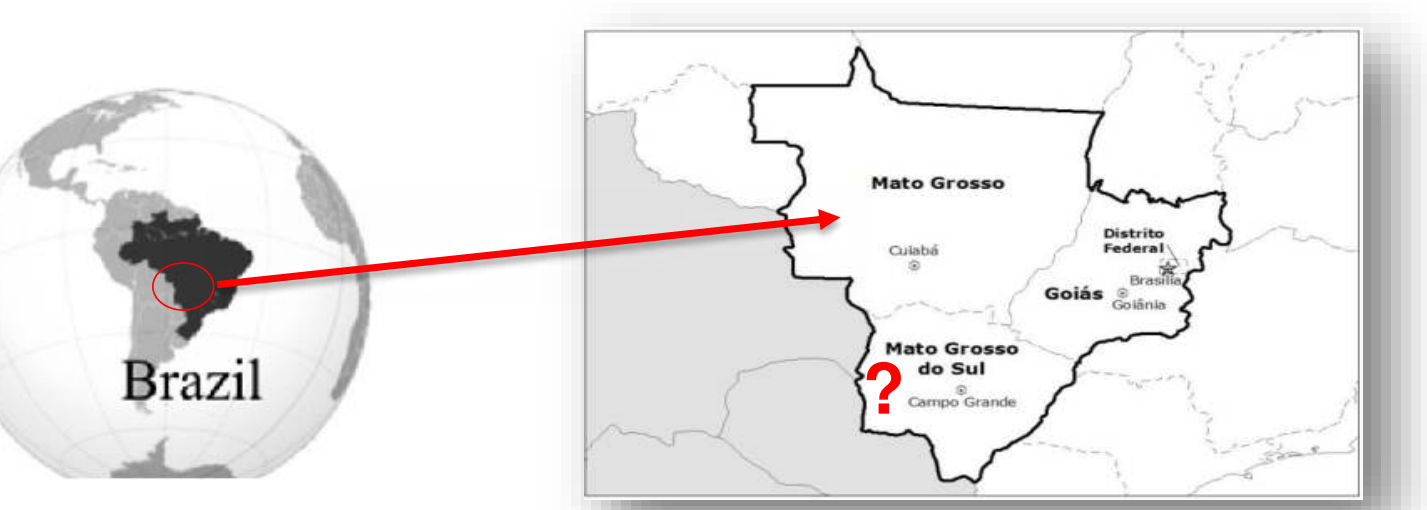

FIGURE 2. Venn diagram shows the percentage of ID positivity Mato Grosso do Sul (MS) patients. The figure shows the low positivity when the serum of the patients is tested with the standard antigen (Pb339) and the high positivity when tested with the P. lutzii antigen. While, Brazil map presents the midwest region, highlighting the MS state, due to lack of studies confirming the $P$. lutzii prevalence
From 43 PCM patients, 33 (76.74\%) were positive on ID, 14 (32.5\%) on direct exam, $30(70 \%)$ on histopathology, while culture had the worst performance with only $4 / 43$ positive patients $(9.3 \%)$.

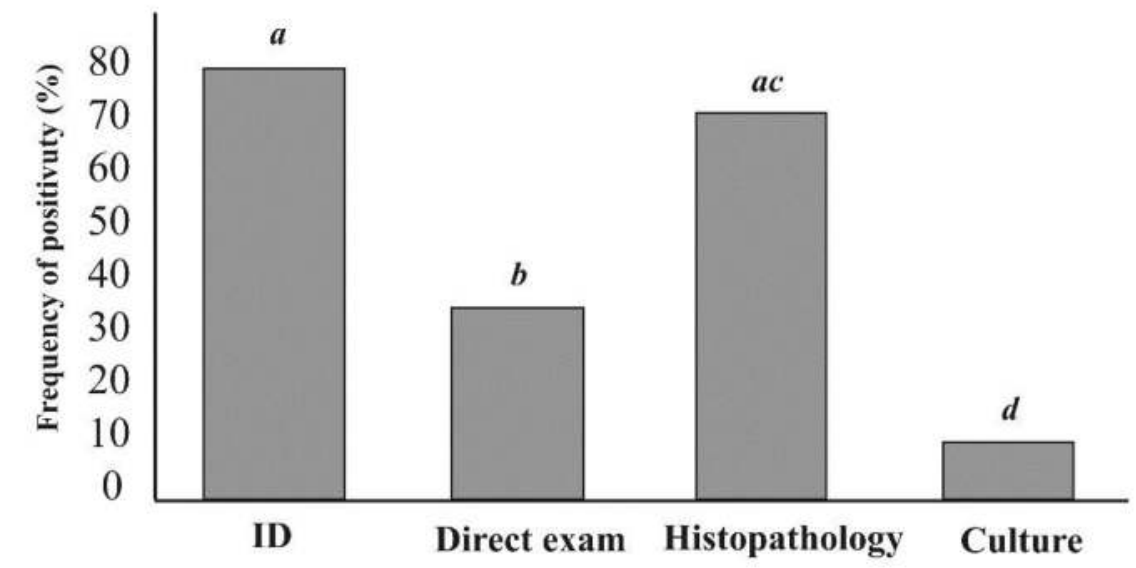

FIGURE 3. The graphic shows the frequency of positivity to test applied to screening PCM. The comparison sanalysis between tests was evaluated by Cochran's Q test. Different letters indicates significant statistical diferences between tests $(\mathrm{p}<0.05)$

A comparative performance of double immunodiffusion (ID), direct exam, histopathology and culture from 43 PCM confirmed patients were conducted using Cochran's Q test (Figure 3), which revealed Q-value $\left(X^{2}\right)$ of 42.43 considering $\mathrm{Q}(3)=7.82, \mathrm{p}<0.05$, indicating that there is difference in performance of tests applied to PCM screening.

Multiple comparisons two-by-two with critical value $(\mathrm{S} \alpha)$ of 2.79 indicates statistical difference between proportions of positivity of culture and ID test $(S=5.49)$, histopathology and culture $(S=5.29)$, direct exam and histopathology $(\mathrm{S}=3.25)$ and direct exam and ID test $(\mathrm{S}=3.46)$.

Finally, when we compare the concordances between antigen from $P$. lutzii and $P$. brasiliensis $(P b-339)$ by ID test using kappa coefficient. Then, we found value of 0.02 , reflecting a weak agreement.

Therefore, this data suggest differences in specificity of ExoAgs obtained from Paracoccidioides species

The molecular identification of the clinical isolates are under progress, what will help us to clarify the PCM reactivity profile at Brazilian Midwest region.

\section{REFERENCES}

Gegembauer, G.; Araújo, L.M.; Pereira, E.F.; Rodrigues, A.M.; Paniago, A.M.M. et al.; (2014) Serology of Paracoccidioidomycosis due to Paracoccidioides lutzii. PLoSNeglTropDis8(7): e2986.

Da Silva Julhiany de Fátima, de Oliveira Haroldo Cesar, Marcos Caroline Maria, Assato Patrícia Akemi. Almeida Ana Marisa Fusco, Mendes-Giannini Maria José Soares, Advances and challenges in Paracoccidioidomycosis serology caused by Paracoccidioides species complex: na update (2015). Diagnostic Microbiology and Infectious Disease.

MuñozJF, FarrerRA, DesjardinsCA, GalloJE, SykesS, SakthikumarS, MisasE, WhistonEA, BagagliE, Soares CMA, Teixeira MDM, Taylor JW, Clay OK, McEwenJG, CuomoCA. Genome diversity, recombination, and virulence across the major lineages of Paracoccidioides (2016). mSphere1(5):e00213-16.

Teixeira MM, Theodoro RC, Nino-Vega G, Bagagli E, Felipe MSS. Paracoccidioides Species Complex: Ecology, Phylogeny, Sexual Reproduction and Virulence. (2014) PLoSPathog10(10): e1004397.

Arantes TD, Theodoro RC, Teixeira MdM, Bosco SdMG, Bagagli E. Environmental Mapping of Paracoccidioides spp. in Brazil Reveals New Clues into Genetic Diversity, Biogeography and Wild Hos Association. (2016) PLoSNegITropDis10(4): e0004606.

Camargo, Z. P. Serology of Paracoccidioidomycosis. Mycopathologia. V.165, n. 4-5, p.289-302, 2008.

\section{PIBIC UFMS} Programa Institucional die
Bolsass

Development Agency: This work was supported by FUNDECT/DECIT-MS/CNPq/SES No 03/2016 - PPSUS-MS. JF and ANRP were recipients of scholarship provided of Universidade Federal do Mato Grosso do Sul / PIBIC/PIBITI/VOLUNTÁRIOS - CNPq/UFMS 2016/2017. SSW received support by CNPq - Apoio à Participação em Eventos Científicos no Exterior - AVG (No 450193/2018-7) and UFMS/PROPP - Participação e Apresentação de Trabalhos de DOCENTES ( $\left.\mathrm{N}^{\circ} 101 / 2018\right)$. 